\title{
Fin de la clause du besoin: une chance à saisir
}

La fin prochaine de la clause du besoin est une bonne occasion pour s'arrêter, réfléchir, tirer un bilan, vérifier les positions et redresser le cap.

Dans ma perception des choses, j'entrevois deux attitudes différentes, dont ni l'une ni l'autre n'est convaincante:

- si le corps médical adopte une attitude de refus et que nous nous cantonnons dans le rôle du «Neinsager», nous ne serons certes pas responsables des décisions prises par nos partenaires, mais nous devons néanmoins les subir;

- si nous laissons à nos partenaires ou à la politique nationale le soin de résoudre un problème urgent, ils ne disposeront pas des connaissances détaillées indispensables et mettront en œuvre des projets unilatéraux discutables et qui n'auront pas mûri.

Dans notre système de santé, pouvons-nous aborder ensemble les questions ouvertes pour les résoudre? Les dernières années nous fournissent à cet égard des exemples convaincants et de qualité:

- l'introduction du TARMED dans le domaine ambulatoire pour les caisses-maladie et les assureurs-accidents - sous garantie de la neutralité des coûts - a réussi, comme d'ailleurs le maintien de la Convention prestations-prix;

- plusieurs directions cantonales de la santé ont, conjointement avec les sociétés de médecine, délivré de manière ciblée des autorisations devenues nécessaires, malgré le blocage de l'accès à la pratique privée;

- dans le cadre des réseaux de médecins, nos consœurs et confrères concluent des conventions partenariales avec les assureurs.
Que signifient ces exemples pour la fin imminente de la clause du besoin?

Le bilan est clair: les objectifs fixés n'ont pas été atteints. Le travail qui aurait dû être fait pour créer une base de régulation loyale et efficace du nombre de médecins n'a pas été réalisé. Trois options se présentent maintenant:

1. La clause du besoin est levée.

2. Si les responsables politiques veulent la maintenir, ils doivent limiter sa prolongation à deux ans. En outre, ils doivent absolument en modifier et en assouplir l'application sans oublier de créer les bases nécessaires à une régulation des fournisseurs de prestations médicales.

3. Si la clause du besoin est maintenue telle quelle, nous ne pourrons pas l'accepter et devrons combattre cette décision.

Dans le document intitulé «L'opinion de la FMH» concernant la levée de la clause du besoin (cf. page 397 de la présente édition), nous présentons nos réflexions sur la régulation de la fourniture de prestations médicales dans le domaine ambulatoire et hospitalier. Nous avons également élaboré des propositions de solutions qu'il s'agit maintenant d'intégrer dans la discussion politique; nous sommes prêts à les développer de manière approfondie.

Le moment est venu de saisir notre chance.

Dr Olivier Kappeler, membre du Comité central de la FMH

Pour une recherche fructueuse dans la liste des médecins de la FMH: 